\title{
DETERMINANTES DO AMBIENTE EDUCACIONAL EM UMA ESCOLA DE FISIOTERAPIA: UMA AVALIAÇÃO PSICOMÉTRICA
}

\author{
DETERMINANTS OF THE EDUCATIONAL ENVIRONMENT IN A \\ PHYSIOTHERAPY SCHOOL: A PSYCHOMETRIC EVALUATION
}

Thaísa Paiva De Oliveira (ORCID: 0000-0003-3866-7954) ${ }^{1}$

Dione Marçal Lima (ORCID: 0000-0003-1248-3479)

Alessandra Vitorino Naghettini (ORCID: 0000-0003-2329-6222)

Autor Correspondente Thaísa Paiva De Oliveira E-mail: thaisa_paiva@yahoo.com.br

${ }^{1}$ Programa de Pós-graduação Stricto Sensu Ensino na Saúde, Universidade Federal de Goiás.

a) Trabalho Acadêmico resultante da dissertação de mestrado de Thaísa Paiva De Oliveira, "O ambiente educacional em um curso de fisioterapia: percepção discente", defendido em 2017 na Universidade Federal de Goiás.

\begin{abstract}
RESUMO
O objetivo do presente estudo foi explorar áreas com aspectos negativos e positivos no ambiente educacional pela percepção dos alunos de graduação em fisioterapia e investigar sua relação com as características demográficas dos entrevistados. Trata-se de um estudo transversal analítico que utilizou o instrumento DREEM (Dundee Ready Education Environment Measure), na perspectiva de cinco dimensões: percepção da aprendizagem, percepção quanto aos docentes, autopercepção do acadêmico, percepção da atmosfera educacional, percepção das relações sociais. Foram avaliados os 50 itens do questionário, interpretados pelo escore, cujo valor máximo geral foi 200 e sua relação com características sociodemográficas. Todos os 232 estudantes matriculados na instituição foram convidados a participar da pesquisa; destes, 179 discentes de fisioterapia $(77,15 \%)$ nos diferentes períodos do curso em $2016 / 1$ responderam ao questionário. A média do escore global da percepção discente sobre o ambiente educacional foi de 126/200. Na avaliação das dimensões, observaram-se os seguintes escores: percepção da aprendizagem, 30/48; percepção quanto aos docentes 28/44; autopercepção do acadêmico, 21/32; percepção da atmosfera educacional, 30/48; e percepção das relações sociais, 17/28. Baseado no escore DREEM Global, o estudo revelou um ambiente educacional mais 'positivo' que 'negativo'. Questões individuais indicaram melhor pontuação em itens como: "ter amigos na faculdade" e "confiança na aprovação das disciplinas". Entretanto, foi destacada pontuação negativa nos itens que se referem a "ausência de apoio a estudantes estressados" e "ênfase na memorização de conteúdos". O instrumento DREEM se mostrou confiável para avaliação do ambiente educacional no curso de fisioterapia, definindo áreas que apresentam maior necessidade de intervenção.
\end{abstract}

Palavras-chave: Avaliação educacional; Educação superior; Fisioterapia.

\begin{abstract}
The aim of the present study was to explore areas with negative and positive aspects in the educational environment from the perception of undergraduate physiotherapy students and to investigate their relationship with the demographic characteristics of respondents. This is an analytical cross-sectional study that used the DREEM (Dundee Ready Education Environment Measure) instrument, from the perspective of five dimensions: perception of learning, perception of teachers, self-perception of the academic, perception of the educational atmosphere, perception of social relations. Fifty items of the questionnaire, interpreted by the score, whose maximum overall value was 200 and their relation with sociodemographic characteristics were evaluated. All 232 students enrolled in the institution were invited to participate in the research, of which 179 physiotherapy students $(77.15 \%)$ in different stages of the course in 2016.1 answered the questionnaire. The average global student perception score on the educational environment was $126 / 200$. In the evaluation of the dimensions, the following scores were observed: perception of learning $30 / 48$, perception of teachers $28 / 44$, self-perception of academic $21 / 32$, perception of educational atmosphere $30 / 48$ and perception of social relationships 17/28. Based on the Global DREEM score, the study revealed a more 'positive' than 'negative' educational environment. Individual questions indicated better scores on items such as "having friends in college" and "confidence in passing subjects". However, negative scores were highlighted on items that refer to "lack of support for stressed students" and "emphasis on memorizing content". The DREEM instrument proved to be reliable for assessing the educational environment in the physiotherapy course, defining areas that are most in need of intervention.
\end{abstract}

Keywords: Educational measurement; Education, Higher; Physical therapy specialty. 


\section{INTRODUÇÃO}

Os discentes do curso de fisioterapia podem apresentar altos índices de ansiedade, influenciando diretamente em sua qualidade de vida. O estresse e a ansiedade desses alunos dentro do ambiente educacional podem influenciar no seu potencial de aprendizagem ${ }^{1}$.

O ambiente educacional é moldado, principalmente, pelas interações entre os diferentes grupos de partes interessadas e as estruturas organizacionais do ambiente. Esse ambiente, ocasionalmente chamado de "clima" ou "atmosfera", é complexo, multifacetado e pode ser descrito como o espírito e a personalidade de uma instituição educacional ${ }^{2}$. Ele influencia na satisfação e no sucesso do discente ${ }^{2}$, uma vez que este pode ter um caráter tanto competitivo, estressante, motivacional quanto passivo e indiferente. Nesse sentido, a Instituição de Ensino deve oferecer um ambiente estimulante para o aluno, com foco na qualidade da aprendizagem, o que pode ser mensurado por meio do feedback dos discentes ${ }^{4}$.

Alguns instrumentos já amplamente utilizados avaliam o ambiente educacional na perspectiva do discente, por meio de métodos qualitativos, quantitativos ou mistos ${ }^{5-7}$.

O Dundee Ready Educational Environment Measure (DREEM) é um método psicométrico DREEM, desenvolvido com base em informações sobre o planejamento curricular, com o intuito de identificar irregularidades no ambiente educacional, validado por Roof et al.8 e traduzido para o português 9 . O DREEM é considerado culturalmente confiável, sendo aplicado em diversos cursos na área da saúde ${ }^{4,5,10}$.

O objetivo do presente estudo foi explorar áreas com aspectos negativos e positivos no ambiente educacional pela percepção dos discentes de graduação em fisioterapia utilizando o instrumento DREEM e investigar sua relação com as características demográficas dos entrevistados, tendo em vista a importância dessa temática e a escassez de estudos sobre o ambiente educacional no curso de fisioterapia.

\section{MÉTODOS}

Trata-se de um estudo transversal e descritivo realizado com discentes do curso de fisioterapia de um Centro Universitário localizado na região Centro-Oeste. O curso é desenvolvido em um modelo tradicional com pouca integração, divido em duas fases: pré-clínica e clínica.

Todos os discentes matriculados no curso de fisioterapia em seus diversos períodos $(\mathrm{n}=232)$ foram convidados a participar da pesquisa. Foram incluídos aqueles regularmente matriculados no ano de 2016/1 que aceitaram participar da pesquisa e assinaram o Termo de Consentimento Livre e Esclarecido (TCLE).

Foram excluídos do estudo discentes que estavam afastados das atividades acadêmicas (licença maternidade, afastamento por motivos pessoais, trancamento de matrícula) ou aqueles que não estavam presentes no dia de aplicação do questionário.

\section{Coleta de Dados}

Foram coletados dados referentes ao questionário DREEM e relacionados com o perfil sociodemográfico dos participantes, incluindo as variáveis: gênero, idade, renda familiar e estágio de estudo (pré-clínico ou clínico). 
A coleta de dados ocorreu de forma presencial, em sala de aula, durante uma hora/aula de 50 minutos no primeiro semestre do ano de 2016. Os discentes foram informados sobre a pesquisa, seus objetivos, orientados sobre o TCLE. Em seguida, preencheram o questionário.

\section{Delineamento do Estudo}

O questionário DREE foi traduzido para o português ${ }^{9}$ e validado por Roff et al. ${ }^{8}$. Possui 50 questões, configuradas em escalas Likert de 5 pontos que variam de 0 , "discorda fortemente", a 5, "concorda fortemente". É comumente interpretado na literatura em forma de escores, sendo o escore máximo geral no valor de 200 . Cada dimensão avalia diferentes aspectos que participam na formação do ambiente educacional.

A dimensão Percepção da Aprendizagem avalia a percepção do discente acerca do ensino, dos métodos de ensino empregados e dos objetivos do curso; possui 12 questões, sendo o escore máximo 48.

A dimensão Percepção quanto aos Docentes avalia a percepção do discente sobre as atitudes e a didática dos docentes do curso; possui 11 questões, sendo o escore máximo 44.

A dimensão Autopercepção do Acadêmico avalia a percepção do discente sobre seu desempenho acadêmico; possui 8 questões, sendo o escore máximo 32.

A dimensão Percepção da Atmosfera Educacional avalia a percepção do discente sobre a atmosfera durante as aulas teóricas e práticas; possui 12 questões, sendo o escore máximo 48.

A dimensão Percepção das Relações Sociais avalia a percepção do discente acerca das suas relações sociais no ambiente acadêmico; possui 7 questões, sendo o escore máximo 28.

Os itens do questionário são assertivas que avaliam o ambiente de maneira positiva. As assertivas número 4, 8, 9, 17 , $25,35,39,48$ e 50 abrangem aspectos negativos; portanto, as pontuações atribuídas a eles foram invertidas ${ }^{5}$.

De Oliveira et al

\section{Análise dos Dados}

Os dados coletados foram, inicialmente, tabulados em uma planilha do Windows Excel ${ }^{\circledR} 2013$; posteriormente, analisados com a utilização do pacote estatístico Statistical Package of Social Sciences (SPSS 23.0).

Neste estudo, aplicou-se o teste Shapiro-Wilk (SW); variáveis não demonstraram normalidade, optando-se por testes não paramétricos.

A confiabilidade do questionário foi testada calculando-se o Coeficiente Alfa de Cronbach $(\alpha)$ que verificou a consistência interna do instrumento para a amostra estudada.

A associação das dimensões do questionário entre período e fases (pré-clínica e clínica) foi testada utilizando os testes de Mann-Whitney. Para este estudo, foi adotado um nível de significância de $5 \%(\mathrm{p} \leq 0,05)$.

Para a análise das questões individuais, utilizaram-se a média e o desvio-padrão dos escores em cada questão. Questões com média $>3,5$ correspondem a pontos muito positivos; pontuação média entre 3 e 3,5 corresponde a pontos positivos; pontuação média entre 2 e 3 corresponde a aspectos que podem ser melhorados; e questões com média $<2$ revelam áreas com dificuldade e, portanto, sinalizam os pontos negativos do ambiente educacional ${ }^{9,11,12}$.

\section{Aspectos Éticos}

O desenvolvimento da pesquisa atendeu às recomendações propostas pelo Conselho Nacional de Saúde, por meio da Resolução no 466/12, e foi aprovado pelo Comitê de Ética em Pesquisa, sob o Protocolo $n^{\circ} 002913 / 2016$. 


\section{RESULTADOS}

Participaram do estudo 179 discentes do curso de fisioterapia que representaram $77,15 \%$ do total dos matriculados em 2016/1.

\section{Avaliação sociodemográfica}

Dos entrevistados, $153(85,47 \%)$ eram do sexo feminino, e 26 (14,53\%), do sexo masculino. Quanto à fase do curso, $120 / 179(67,03 \%)$ se encontravam na fase pré-clínica, enquanto 59/179 $(32,96 \%)$ estavam na fase clínica. A faixa etária média foi de $22,76( \pm 5,73)$ anos de idade. A renda familiar foi menor que três salários mínimos entre $120(67,04)$ dos entrevistados.
Avaliação do meio ambiente educacional

O escore global encontrado foi de 126 pontos de um total máximo de 200 . O valor de Alfa de Cronbach ( $\alpha$ ), para avaliação da confiabilidade do instrumento, foi de 0,87 para o escore geral.

\section{Dimensões do DREEM}

\section{Dimensão 1: Percepção da Aprendizagem (PA)}

Essa dimensão apresentou média dos escores de 27,3 (máximo: 48). Destacou-se como ponto positivo o conhecimento dos discentes quanto aos objetivos do curso. Como ponto negativo, observou-se um ambiente que incentiva o aprendizado por meio da memorização e ensino centrado na figura do professor. Questões com escore entre 2 e 3 representaram $75 \%$ do total. Na tabela 1, estão descritas as questões ordenadas conforme o escore.

Tabela 1. Escores atingidos na dimensão Percepção da Aprendizagem

\begin{tabular}{|c|c|c|c|}
\hline Questões & $\begin{array}{c}\text { Média } \\
\text { Escores }\end{array}$ & $\begin{array}{l}\text { Desvio- } \\
\text { padräo }\end{array}$ & Percepção \\
\hline $\begin{array}{l}\text { Dimensão } 1 \text { - PA (escore } \\
\text { máximo: } 48 \text { pontos) }\end{array}$ & 27,34 & & $\begin{array}{c}\text { Mais positiva que } \\
\text { negativa }\end{array}$ \\
\hline $\begin{array}{l}\text { 25. O ensino enfatiza muito } \\
\text { o aprendizado de fatos } \\
\text { memorizáveis }\end{array}$ & 1,58 & 0,86 & Ponto negativo \\
\hline $\begin{array}{l}\text { 48. O ensino é muito } \\
\text { centrado no professor }\end{array}$ & 1,60 & 0,93 & Ponto negativo \\
\hline $\begin{array}{l}\text { 13. O ensino é centrado no } \\
\text { estudante } \\
\text { autoaprendizado) }\end{array}$ & 2,38 & 0,92 & $\begin{array}{l}\text { Pode ser } \\
\text { melhorado }\end{array}$ \\
\hline $\begin{array}{l}\text { 7. O ensino adotado é } \\
\text { frequentemente } \\
\text { estimulante }\end{array}$ & 2,45 & 0,98 & $\begin{array}{l}\text { Pode ser } \\
\text { melhorado }\end{array}$ \\
\hline $\begin{array}{l}\text { 22. O método de ensino se } \\
\text { preocupa em desenvolver } \\
\text { minha confiança }\end{array}$ & 2,56 & 1,03 & $\begin{array}{l}\text { Pode ser } \\
\text { melhorado }\end{array}$ \\
\hline $\begin{array}{l}\text { 24. O tempo para ensino é } \\
\text { bem utilizado }\end{array}$ & 2,63 & 1,03 & $\begin{array}{l}\text { Pode ser } \\
\text { melhorado }\end{array}$ \\
\hline $\begin{array}{l}\text { 20. O ensino é bastante } \\
\text { focado e coeso }\end{array}$ & 2,64 & 0,94 & $\begin{array}{l}\text { Pode ser } \\
\text { melhorado }\end{array}$ \\
\hline $\begin{array}{l}\text { 16. O ensino se preocupa } \\
\text { em desenvolver minha } \\
\text { competência }\end{array}$ & 2,77 & 0,97 & $\begin{array}{l}\text { Pode ser } \\
\text { melhorado }\end{array}$ \\
\hline $\begin{array}{l}\text { 47. A importância da } \\
\text { educação continuada é } \\
\text { enfatizada }\end{array}$ & 2,83 & 0,89 & $\begin{array}{l}\text { Pode ser } \\
\text { melhorado }\end{array}$ \\
\hline $\begin{array}{l}\text { 44. O ensino me encoraja } \\
\text { a buscar meu próprio } \\
\text { aprendizado }\end{array}$ & 2,85 & 0,85 & $\begin{array}{l}\text { Pode ser } \\
\text { melhorado }\end{array}$ \\
\hline $\begin{array}{l}\text { 38. Tenho certeza sobre os } \\
\text { objetivos deste curso }\end{array}$ & 3,05 & 0,83 & Ponto forte \\
\hline
\end{tabular}

PA: Percepção da Aprendizagem. 
Dimensão 2: Percepção quanto aos Docentes (PD)

Nessa dimensão, a média de escore foi de 28,3 (máximo: 44). Observaram-se como aspectos negativos os sinais de irritabilidade dos docentes com os estudantes. Questões com escore entre 2 e 3 representam $91 \%$, conforme indicado na tabela 2 .

Tabela 2. Escores atingidos na dimensão Percepção quanto aos Docentes

\begin{tabular}{|c|c|c|c|}
\hline Questões & $\begin{array}{c}\text { Média } \\
\text { Escores }\end{array}$ & $\begin{array}{l}\text { Desvio- } \\
\text { padräo }\end{array}$ & Percepção \\
\hline $\begin{array}{l}\text { Dimensão } 2-\text { PD (escore } \\
\text { máximo: } 44 \text { pontos) }\end{array}$ & 28,27 & & Na direção certa \\
\hline $\begin{array}{l}50 \text {. Os estudantes irritam os } \\
\text { professores }\end{array}$ & 1,87 & 1,27 & Ponto negativo \\
\hline $\begin{array}{l}\text { 9. Os professores são } \\
\text { autoritários }\end{array}$ & 2,11 & 1,19 & $\begin{array}{l}\text { Pode ser } \\
\text { melhorado }\end{array}$ \\
\hline $\begin{array}{l}\text { 39. Os professores ficam } \\
\text { nervosos em sala de aula }\end{array}$ & 2,38 & 1,21 & $\begin{array}{l}\text { Pode ser } \\
\text { melhorado }\end{array}$ \\
\hline $\begin{array}{l}\text { 29. Os professores dão um } \\
\text { bom feedback aos estudantes }\end{array}$ & 2,53 & 0,95 & $\begin{array}{l}\text { Pode ser } \\
\text { melhorado }\end{array}$ \\
\hline $\begin{array}{l}\text { 32. Os professores nos dão } \\
\text { criticas construtivas }\end{array}$ & 2,59 & 0,96 & $\begin{array}{l}\text { Pode ser } \\
\text { melhorado }\end{array}$ \\
\hline $\begin{array}{l}\text { 2. É possivel entender os } \\
\text { professores em suas aulas }\end{array}$ & 2,68 & 0,87 & $\begin{array}{l}\text { Pode ser } \\
\text { melhorado }\end{array}$ \\
\hline $\begin{array}{l}\text { 37. Os professores dão } \\
\text { exemplos muito claros }\end{array}$ & 2,74 & 0,91 & $\begin{array}{l}\text { Pode ser } \\
\text { melhorado }\end{array}$ \\
\hline $\begin{array}{l}\text { 6. Professores têm se } \\
\text { mostrado pacientes com os } \\
\text { pacientes }\end{array}$ & 2,76 & 1,02 & $\begin{array}{l}\text { Pode ser } \\
\text { melhorado }\end{array}$ \\
\hline $\begin{array}{l}\text { 40. Os professores são bem } \\
\text { preparados para as aulas }\end{array}$ & 2,86 & 0,85 & $\begin{array}{l}\text { Pode ser } \\
\text { melhorado }\end{array}$ \\
\hline $\begin{array}{l}\text { 8. Os professores ridicularizam } \\
\text { os estudantes }\end{array}$ & 2,87 & 1,19 & $\begin{array}{l}\text { Pode ser } \\
\text { melhorado }\end{array}$ \\
\hline $\begin{array}{l}\text { 18. Os professores } \\
\text { conseguem se comunicar bem } \\
\text { com os pacientes }\end{array}$ & 2,88 & 0,84 & $\begin{array}{l}\text { Pode ser } \\
\text { melhorado }\end{array}$ \\
\hline
\end{tabular}

PD: Percepção quanto aos Docentes. 
Dimensão 3: Autopercepção do Acadê$\operatorname{mico}(A A)$

Essa dimensão apresentou média de escore de 20,9 (máximo: 32). Os discentes revelaram como ponto positivo a importância do conteúdo para o curso e a confiança na aprovação. Como ponto negativo, foi identificada a forma de estudar do estudante. Questões com escore entre 2 e 3 representaram $62,5 \%$, conforme indicado na tabela 3 .

Tabela 3. Escores atingidos na dimensão Autopercepção do Acadêmico

\begin{tabular}{|c|c|c|c|}
\hline Questões & $\begin{array}{c}\text { Média } \\
\text { Escores }\end{array}$ & Desvio-padrão & Percepção \\
\hline $\begin{array}{l}\text { Dimensão } 3- \\
\text { (escore máximo: } \\
\text { pontos) }\end{array}$ & 20,89 & & $\begin{array}{c}\text { Mais aspectos } \\
\text { positivos que } \\
\text { negativos }\end{array}$ \\
\hline $\begin{array}{l}\text { 5. Como estudava } \\
\text { antes também } \\
\text { funciona neste curso }\end{array}$ & 1,78 & 1,18 & Ponto negativo \\
\hline $\begin{array}{l}27 . \quad \text { Tenho boa } \\
\text { capacidade } \\
\text { memória para tudo que } \\
\text { preciso }\end{array}$ & 2,29 & 1,06 & $\begin{array}{l}\text { Pode ser } \\
\text { melhorado }\end{array}$ \\
\hline 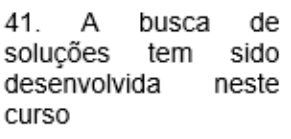 & 2,35 & 1,02 & $\begin{array}{l}\text { Pode ser } \\
\text { melhorado }\end{array}$ \\
\hline $\begin{array}{l}\text { 26. O ensino do ano } \\
\text { anterior me preparou } \\
\text { bem para este ano }\end{array}$ & 2,50 & 1,06 & $\begin{array}{l}\text { Pode ser } \\
\text { melhorado }\end{array}$ \\
\hline $\begin{array}{l}\text { 21. Sinto que venho } \\
\text { sendo preparado para } \\
\text { a profissão }\end{array}$ & 2,69 & 1,00 & $\begin{array}{l}\text { Pode ser } \\
\text { melhorado }\end{array}$ \\
\hline $\begin{array}{lr}31 . \quad \text { Aprendi } & \text { muito } \\
\text { sobre relacionamento } \\
\text { pessoal } & \text { nesta } \\
\text { profissão } & \end{array}$ & 2,83 & 1,06 & $\begin{array}{l}\text { Pode ser } \\
\text { melhorado }\end{array}$ \\
\hline 45. Muito do que tenho & & & \\
\hline \begin{tabular}{llr} 
visto & \multicolumn{2}{c}{ parece } \\
importante & para a \\
fisioterapia &
\end{tabular} & 3,21 & 0,77 & Ponto forte \\
\hline $\begin{array}{l}\text { 10. Estou confiante } \\
\text { que vou ser aprovado } \\
\text { neste ano }\end{array}$ & 3,24 & 0,97 & Ponto forte \\
\hline
\end{tabular}

AA: Autopercepção do Acadêmico. 


\section{Dimensão 4: Percepção da Atmosfera Educacional (PAE)}

Nessa dimensão, a média de escore foi de 30,1 (máximo: 48). Como aspecto negativo, destacou-se a prática dos estudantes em colar durante as provas. Questões com escore entre 2 e 3 representaram $62,5 \%$, conforme indicado na tabela 4 .

Tabela 4. Escores atingidos na dimensão Percepção da Atmosfera Educacional

\begin{tabular}{|c|c|c|c|}
\hline Questões & $\begin{array}{c}\text { Média } \\
\text { Escores }\end{array}$ & Desvio-padrão & Percepção \\
\hline $\begin{array}{lcr}\text { Dimensão } & 4- & \text { PAE } \\
\text { (escore máximo: } & 48 \\
\text { pontos) } & & \end{array}$ & 30,06 & & $\begin{array}{c}\text { Mais aspectos } \\
\text { positivos que } \\
\text { negativos }\end{array}$ \\
\hline $\begin{array}{l}\text { 17. A prática de colar em } \\
\text { provas é comum nesta } \\
\text { faculdade }\end{array}$ & 1,82 & 1,28 & Ponto negativo \\
\hline $\begin{array}{l}\text { 12. Esta faculdade é } \\
\text { bastante pontual nos } \\
\text { cursos }\end{array}$ & 2,26 & 1,01 & $\begin{array}{l}\text { Pode ser } \\
\text { melhorado }\end{array}$ \\
\hline $\begin{array}{l}34 \text { O ambiente é } \\
\text { tranquilo durante os } \\
\text { seminários }\end{array}$ & 2,38 & 1,02 & $\begin{array}{l}\text { Pode ser } \\
\text { melhorado }\end{array}$ \\
\hline $\begin{array}{l}\text { 11. O ambiente é } \\
\text { tranquilo durante as } \\
\text { aulas no estágio }\end{array}$ & 2,43 & 0,86 & $\begin{array}{l}\text { Pode ser } \\
\text { melhorado }\end{array}$ \\
\hline $\begin{array}{l}23 . \quad \mathrm{O} \text { ambiente é } \\
\text { tranquilo durante as } \\
\text { aulas }\end{array}$ & 2,43 & 1,06 & $\begin{array}{l}\text { Pode ser } \\
\text { melhorado }\end{array}$ \\
\hline $\begin{array}{l}\text { 43. O ambiente me } \\
\text { estimula a aprender }\end{array}$ & 2,53 & 0,97 & $\begin{array}{l}\text { Pode ser } \\
\text { melhorado }\end{array}$ \\
\hline $\begin{array}{lr}36 . \quad \text { Tenho } & \text { boa } \\
\text { capacidade } & \text { de } \\
\text { concentração } & \end{array}$ & 2,56 & 0,96 & $\begin{array}{l}\text { Pode ser } \\
\text { melhorado }\end{array}$ \\
\hline $\begin{array}{l}\text { 33. Sinto-me confortável } \\
\text { nas aulas }\end{array}$ & 2,60 & 0,98 & $\begin{array}{l}\text { Pode ser } \\
\text { melhorado }\end{array}$ \\
\hline $\begin{array}{l}\text { 49. Sinto-me à vontade } \\
\text { para perguntar o que } \\
\text { quero nas aulas }\end{array}$ & 2,61 & 1,16 & $\begin{array}{l}\text { Pode ser } \\
\text { melhorado }\end{array}$ \\
\hline $\begin{array}{l}\text { 30. Tenho oportunidade } \\
\text { de desenvolver prática } \\
\text { der relacionamento } \\
\text { pessoal }\end{array}$ & 2,74 & 0,95 & $\begin{array}{l}\text { Pode ser } \\
\text { melhorado }\end{array}$ \\
\hline $\begin{array}{l}35 \text {. Tenho achado } \\
\text { minha experiência aqui } \\
\text { desapontadora }\end{array}$ & 2,85 & 1,10 & $\begin{array}{l}\text { Pode ser } \\
\text { melhorado }\end{array}$ \\
\hline $\begin{array}{l}\text { 42. A satisfação é maior } \\
\text { do que o estresse de } \\
\text { estudar fisioterapia }\end{array}$ & 2,85 & 1,07 & $\begin{array}{l}\text { Pode ser } \\
\text { melhorado }\end{array}$ \\
\hline
\end{tabular}

PAE: Percepção da Atmosfera Educacional. 
Essa dimensão apresentou média de escore de 17,5 (máximo: 28). Destacaram-se como pontos positivos a socialização entre os discentes e o fato de se sentirem confortáveis em seu ambiente domiciliar. Como aspecto negativo, os discentes perceberam a necessidade de um programa de apoio aos estudantes que se sentem estressados. Questões com escore entre 2 e 3 representaram $42,8 \%$, conforme a tabela 5 .

Tabela 5. Escores atingidos na dimensão Percepção das Relações Sociais

\begin{tabular}{|c|c|c|c|}
\hline Questões & $\begin{array}{c}\text { Média } \\
\text { Escores }\end{array}$ & Desvio-padrão & Percepção \\
\hline $\begin{array}{lcr}\text { Dimensão } & 5- & \text { PRS } \\
\text { (escore máximo: } & 28 \\
\text { pontos) } & & \end{array}$ & 17,50 & & Não é tão ruim \\
\hline $\begin{array}{l}\text { 3. Existe um bom } \\
\text { programa de apoio para } \\
\text { estudantes estressados }\end{array}$ & 0,82 & 0,99 & Ponto negativo \\
\hline $\begin{array}{l}\text { 14. Raramente me sinto } \\
\text { desestimulado neste } \\
\text { curso }\end{array}$ & 2,00 & 1,23 & Ponto negativo \\
\hline $\begin{array}{l}\text { 4. Tenho estado muito } \\
\text { cansado para aproveitar } \\
\text { este curso }\end{array}$ & 2,32 & 1,27 & $\begin{array}{l}\text { Pode ser } \\
\text { melhorado }\end{array}$ \\
\hline $\begin{array}{l}\text { 28. Raramente me sinto } \\
\text { sozinho }\end{array}$ & 2,44 & 1,23 & $\begin{array}{l}\text { Pode ser } \\
\text { melhorado }\end{array}$ \\
\hline $\begin{array}{l}\text { 19. Minha vida social é } \\
\text { boa }\end{array}$ & 3,20 & 0,81 & Ponto forte \\
\hline $\begin{array}{l}\text { 46. Moro em um lugar } \\
\text { confortável }\end{array}$ & 3,32 & 0,78 & Ponto forte \\
\hline $\begin{array}{l}\text { 15. Tenho bons amigos } \\
\text { na faculdade }\end{array}$ & 3,45 & 0,77 & Ponto forte \\
\hline
\end{tabular}

PRS: Percepção das Relações Sociais. 


\section{DISCUSSÃO}

Analisando o presente estudo, observou-se uma percepção geral do discente, mais positiva que negativa (Escore global 126/200). Na literatura, identificamos apenas um curso de fisioterapia já avaliado, em que os discentes o classificaram como ambiente educacional "mais positivo que negativo", com escore em nível de 150/200, tendo como base a percepção da atmosfera e das relações sociais principalmente ${ }^{13}$. Estudos na área da saúde que empregaram o DREEM ${ }^{5,10,12,13}$ mostram escores mais altos entres discentes dos cursos de odontologia e medicina ${ }^{14}$.

A análise dos resultados da dimensão 1 (PA) aponta uma avaliação negativa da percepção do discente quanto à aprendizagem estar associada ao ensino centrado na figura do docente, uma vez que utilização de metodologias ativas usualmente tem recebido maior escore, como relatado em outros estudos ${ }^{4,15,16}$.

A aprendizagem por intermédio da memorização e o ensino centrado no docente, detectados na dimensão 1 (PA), revelam a característica ensino tradiciona dessa instituição. A relação estabelecida entre discentes e docentes, nesse modelo, é hierárquica; a aquisição de conhecimento está associada à memorização do conteúdo estabelecida pelo docente; e mesmo que haja ampla informação ao discente, há formação de um indivíduo passivo ${ }^{17}$.

Identifica-se fragilidade quanto às relações interpessoais no ambiente educacional no presente estudo, como observado na dimensão 2 (PD). A conduta docente contribui para a motivação discente e sua dedicação ao aprendizado ${ }^{19}$, além disso, a fragilidade na relação discente-docente pode ter influenciado os escores da dimensão PA.

O hábito de colar foi considerado comum entre os discentes, observado na dimensão 4 (PAE), demonstrando grande desafio introduzir a ética entre os discentes no ambiente de ensino ${ }^{5,19}$. Da mesma forma, os discentes também revelam a presença do estresse em suas atividades na universidade, o que foi demonstrado na dimensão 5 (PRS), com escore 0,82 .
$\mathrm{O}$ estresse pode estar relacionado com as diferentes fases da trajetória acadêmica, visto que as adequações no início do curso são consideradas fatores estressantes, assim como a projeção e as incertezas em relação ao mercado de trabalho ao final do curso $^{20}$.

Não foi detectado um programa de suporte a estudantes com sinais de estresse na dimensão 5 (PRS), o que pode ser determinante para a baixa pontuação nessa dimensão PRS, problema esse vigente em diversos estudos referentes a várias instituições em diferentes países tais como Paquistão, Irã, Nepal, Nigéria, Arábia Saudita, Marrocos, Turquia, Grécia, Suécia, Portugal, Chile, Kuwait, Índia e Brasil $1^{7,10-12,16,22-24}$.

Ainda nessa dimensão (PRS), destaca-se como ponto positivo o bom relacionamento entre os pares, além da vida social agradável e qualidade do local de moradia. As atividades sociais aparecem como mecanismo de diminuição do estresse tornando o ambiente mais positivo10. Essas percepções podem revelar aspectos comuns de cidades do interior, normalmente pequenas e acolhedoras o suficiente para o discente ter contato com os colegas de sala em outras ocasiões que não estejam restritas ao ambiente educacional.

Observou-se que a percepção dos discentes neste estudo corrobora outro estudo, ambos apresentam percepções mais positivas na dimensão PA no ciclo pré-clínico; percepções mais positivas na dimensão PD no ciclo clínico; percepções mais positivas na dimensão AA no ciclo clínico; percepções mais positivas na dimensão PAE no ciclo pré-clínico; percepções mais positivas na dimensão PRS no ciclo pré-clínico ${ }^{10}$.

É necessário um estudo mais aprofundado quanto à avaliação positiva sobre a certeza de ser aprovado, visto que esse fato reflete um aspecto negativo do ensino.

Algumas limitações foram percebidas neste estudo, as quais podem estar relacionadas com a maior participação de discentes do ciclo pré-clínico comparados com o ciclo específico e o fato de não termos estudado a mobilidade do discente como fator de influência no desgaste acadêmico. $\mathrm{O}$ instrumento também 
possui questões relativas a condutas sobre a prática nos estágios, e os discentes dos primeiros períodos podem ter uma visão subjetiva, cercada de expectativas.

\section{CONCLUSÕES}

O instrumento DREEM se mostrou confiável para avaliação do ambiente educacional no curso de fisioterapia; nesse caso, com uma visão positiva na perspectiva discente. Aspectos individuais positivos foram reforçados pelo apoio de amigos, e aspectos negativos foram observados pela falta de auxílio aos discentes com algum nível de estresse e a ênfase na memorização de conteúdos.

\section{Conflito de Interesses}

Oliveira TP, Lima DM e Naghettini AV declaram que não há conflito de interesse.

\section{AGRADECIMENTOS}

Os autores deste estudo agradecem a participação dos discentes de fisioterapia e da Instituição de Ensino que colaboraram significativamente para a concretização da pesquisa.

\section{REFERÊNCIAS}

1. Costa KLF, Simóes LO, Souza NFL, Andrade VSM, Chaves CMCM, Lopes RA. Avaliação dos níveis de ansiedade, estresse e qualidade de vida em acadêmicos de Fisioterapia. Fisioter Bras. 2019;20(5):659-67.

2. Holt MC, Roff S. Development and validation of the Anaesthetic Theatre Educational Environment Measure (ATEEM). Med Teach. 2004;26:553-58.
3. Hinrichis CP, Ortiz LE, Pérez CE. Relación entre el Bienestar Académico de Estudiantes de Kinesiología de una Universidad Tradicional de Chile y su Percepción del Ambiente Educacional. Formación Universitaria, 2016;9(1):109-16.

4. Chandran CR, Ranjan R. Students' perceptions of educational climate in a new dental college using the DREEM tool. Adv Med Educ Pract. 2015;6:83-92.

5. Fuenzalida B, Pizarro M, Fuentes J, San-Martín C, Rojas V, López-Fuenzalida A, et al. Percepción del ambiente educacional en estudiantes de pregrado de la carrera de Kinesiología: metodología mixta. Educ Med, 2018;380:1-10.

6. Kelly M, Bennett D, Muijtjens A, O'Flynn S, Dornan T. Can less be more? Comparison of an 8-item placementquality measure with the 50-item Dundee Ready Educational Environment Measure (DREEM). Adv Health Sci Educ Theory Pract. 2015;20(4):1027-32. DOI: $10.1007 /$ s10459-015-9582-4.

7. ENNS SC. Avaliação da percepção do ambiente de ensino e sua relação com a qualidade de vida em estudantes de medicina [tese]. São Paulo (SP): Universidade de São Paulo - USP/SP; 2014.

8. Roff S, Mcaleer S, Harden RM, Al-Qahtani M, Ahmed AU, Deza H, et al. Development and validation of the Dundee Ready Education Environment Measure (DREEM). Med Teach. 1997;19(4): 295-9.

9. Vieira JE, Nunes MPT, Martins MA. Directing student response to early patient contact by questionary. BMC Med Educ. 2003;37:119-25.

10. Memon AR, Ali B, Kiyani MM, Ahmed I, Memon A-u-R, Feroz J. Physiotherapy and pharmacy student's perception of educational environment in a medical university from Pakistan. J Pak Med Assoc. 2018;68(1):71-5.

11. Andalib MM, Malekzadeh MM, Aghrahimi Z, Darybeigi M, Yaghmaei B, Ashrafi M-R, et al. Evaluation of Educational Environment for Medical Students of a Tertiary Pediatric Hospital in Tehran, Using DREEM Questionnaire. Iran J 
Pediatr. 2015;25(5):1-7.

12. Roff S, Mcaleer S, Ifere OS, Bhattcharya S. A global diagnostic tool for measuring educational environment: comparing Nigeria and Nepal. Med Teach. 2001;23(4):378-382.

13. Palmagren PJ, Lindquist I, Sundberg T, Nilsson GH, Laksov KB. Exploring perceptions of the educational environment among undergraduate physiotherapy students. Int J Med Educ. 2014;5:13546 ISSN: 2042-6372. DOI: $10.5116 /$ ijme.53a5.7457

14. Vaughan B, Carter A, Macfarlane C, Morrison T. The DREEM, part 1: measurement of the educational environment in an osteopathy teaching program. BMC Med Educ. 2014;14:2-11.

15. Rodríguez-Hopp MP, Providell SG, Castillo CM, Rondanelli BM, Rebolledo J. Análisis del ambiente educacional en escuela de odontología chilena. Rev Clin Periodoncia Implantol Rehabil Oral. 2016;9(2):153-62.

16. Karim J, Al-Halibi B, Marwan Y, Sadeq H, Dawas A, Al-Abdulrazzaq D. The educational environment of the undergraduate medical curriculum at Kuwait University. Adv Med Educ Pract . 2015;6:297-303.

17. Varela DSS, Barbosa MUF, Machado MFAS. Desafios da prática pedagógica no ensino superior. Revista Expressão Católica. 2016;5(1):97-103.

18. Reisdoefer DN, Teixeira EMM, Ramos MG. A influência da relação professor-estudante na aprendizagem discente: percepções de professores de Ciências e Matemática. Revista Exitus. 2017;7(3):64-87.

19. Sousa RN, Conti VK, Salles AA, Mussel ICR. Desonestidade acadêmica: reflexos na formação ética dos profissionais de saúde. Rev Bioét. 2016:24(3):459-68.

20. Murakami S, Panúncio-Pinto MP, Santos JLF, Troncon LEA. Estresse psicológico em estudantes de cursos de graduação da área da saúde: subsídios para promoção de saúde mental. Rev Med. 2019;98(2):108-13.
21. Pereira EMA, Heinzle MRS, Pinto MM. Internacionalização na educação superior e mobilidade estudantil: o vai e vem dos jovens acadêmicos. Revista Espaço Pedagógico. 2017;24(1):35-49.

22. Guimarães AC, Falbo GH, Menezes T, Falbo A. Percepção do Estudante de Medicina acerca do Ambiente Educacional Utilizando o DREEM. Rev Bras Educ Méd. 2015;39(4):517-26.

23. Ahmad MS, Bhayat A, Fadel HT, Mahrous MS. Comparing dental students' perceptions of their educational environment in Northwestern Saudi Arabia. Saudi Med J. 2015;36(4):477-83.

24. Belayachi J, Razine R, Boufars A, Saadi, A, Abouqall R. Moroccan medical students' perceptions of their educational environment. J Educ Eval Health Prof. 2015;12:1-4.
Recebido: 07/01/2020

Aprovado: 06/07/2021 